\title{
Solid State Lasers for Stereolithography
}

\author{
Jouni P. Partanen \\ 3D Systems, Inc. \\ 26081 Avenue Hall, California, 91355
}

\begin{abstract}
Stereolithography is a Rapid Prototyping \& Manufacturing (RP\&M) technique which is used to produce 3 dimensional plastic parts directly out computer files generated by CAD. Stereolithography systems use ultraviolet lasers to solidify liquid resin into the desired form as defined by the $\mathrm{CAD}$ file. In recent years, solid state laser technology has reached the point where it can be applied to stereolithography (SL). Frequency tripling of the powerful lines of diode pumped $\mathrm{Nd} / \mathrm{YAG}, \mathrm{Nd} / \mathrm{YVO}_{4}$, and $\mathrm{Nd} / \mathrm{YLF}$ lasers results in wavelengths of $355 \mathrm{~nm}$ (YAG, $\mathrm{YVO}_{4}$ ), $351 \mathrm{~nm}$ (YLF), and $349 \mathrm{~nm}$ (YLF). All these lines are applicable for SL. Commercial diode pumped lasers with tens or even hundreds milliwatts in the ultraviolet are already available. Efficient frequency tripling of the $\mathrm{Nd} / \mathrm{YAG}, \mathrm{Nd} / \mathrm{YVO}_{4}$, or $\mathrm{Nd} / \mathrm{YLF}$ lasers requires pulsed operation. The pulse repetition rates at maximum average power are typically between 1 and $30 \mathrm{kHz}$. This talk describes the issues relating to the applications of pulsed all solid state lasers in SL.
\end{abstract}

\section{INTRODUCTION}

Stereolithography (SL) is a Rapid Prototyping \& Manufacturing (RP\&M) technique which is used to produce 3 dimensional plastic parts directly out of files generated by Computer Aided Design (CAD) programs. RP\&M systems build the 3-dimensional objects by first converting the $\mathrm{CAD}$ file data into a form of successive 2-dimensional slices. These slices (thin layers) are then formed and joined to 3-dimensional parts using many different methods. In stereolithography, these slices are formed by solidifying a thin layer on the surface of photocurable resin vat using an ultraviolet (uv) laser. The uv laser beam is focused from above to the surface of the vat and the area to be solidified is scanned by two fast moving mirrors - one for each horizontal direction. A new layer of resin is solidified directly above the previously solidified layer attaching immediately to all of the layers below it. Thus the part grows inside the liquid photopolymer vat.

Lasers are today about 35 year old technology. Since the beginning there has been applications of lasers in many different fields. During the last 10 years, the laser technology has reached the large consumer market through lasers in CD players and in fiberoptic communications. These high volume applications use diode lasers which are made using semiconductor technology. These lasers are inexpensive, efficient and reliable. Many other technologies that apply lasers are also considering the use of diode lasers for the same three reasons. Unfortunately, diode lasers are not available at the ultraviolet wavelengths used in 
stereolithography. However, diode lasers can be used to pump other solid state lasers to in order to achieve all solid state laser system for the ultraviolet wavelengths. Although these laser systems are rather complicated and thus not that inexpensive, they can both more reliable and efficient than the gas laser technology presently used in SL. In addition, diode laser pumped solid state lasers for ultraviolet can be made much smaller than the gas lasers at the same power level.

\section{OPTIMAL WAVELENGTH}

Commercially available photopolymer initiators have their optimal absorption at certain specific wavelengths. Laser beams at wavelengths shorter than $300 \mathrm{~nm}$ are not usually suitable for SL because photons at that energy are absorbed by all molecules of the resin. The photons absorbed by other molecules than the photoinitiators do not cause polymerization. In the wavelength range $310-355 \mathrm{~nm}$ the pool of efficient initiator molecules is largest[1]. Thus almost all commercial SL systems use HeCd lasers at $325 \mathrm{~nm}$ or Argon Ion lasers at $351 \mathrm{~nm}$. The photoinitiator molecule absorption cross sections $\sigma$ are usually very different at the wavelengths of $325 \mathrm{~nm}$ and $351 \mathrm{~nm}$. Therefore the photopolymer resins are different for SLA systems with either $\mathrm{HeCd}$ or Ar Ion lasers. Because the absorption cross sections $\sigma$ are usually larger at $325 \mathrm{~nm}$ than at $351 \mathrm{~nm}$ less photoinitiator is needed, and the wavelength around $325 \mathrm{~nm}$ is preferred for stereolithography. HeCd lasers generally provide up to $40 \mathrm{~mW}$ of power at 325 $\mathrm{nm}$ for the SLA-250 (250 $\mathrm{mm} \times 250 \mathrm{~mm}$ build area) and Argon Ion lasers can run up to $600 \mathrm{~mW}$ of power at $351 \mathrm{~nm}$ for the SLA-500 $(500 \mathrm{~mm} \times 500 \mathrm{~mm}$ build area). The average lifetimes of both HeCd lasers and ultraviolet Argon Ion lasers have been extended beyond 4000 hours in the last couple of years.

\section{POSSIBLE SOLID STATE LASERS}

There are at least 3 different possible approaches for all solid state ultraviolet lasers for stereolithography: 1) direct diode laser, 2) frequency doubled diode laser, and 3) frequency converted diode pumped solid state laser.

As mentioned in the introduction direct ultraviolet diode lasers are not yet available. Red diode lasers at $630 \mathrm{~nm}$ are the shortest wavelength lasers that are commercially available. In the last couple of years, the lifetimes of blue lasers have improved beyond 100 hours and more research has been directed towards ultraviolet diode laser technology. However, commercial uv diode lasers are probably 5 or even 10 years away from today.

Red $(630-690 \mathrm{~nm})$ diode laser technology has developed significantly in the last two years. Frequency doubling of $670 \mathrm{~nm}$ laser leads to wavelength of $335 \mathrm{~nm}$, close to optimal for SL. Reasonable high power $(>0.5 \mathrm{~W})$ single mode diodes lasers are already commercially available and frequency doubled versions could be developed in few years.

Frequency converted ultraviolet diode pumped solid state lasers are commercially available in many different form. Frequency tripling of the powerful lines of $\mathrm{Nd} / \mathrm{YAG}$, $\mathrm{Nd} / \mathrm{YVO}_{4}$, and $\mathrm{Nd} / \mathrm{YLF}$ lasers results in wavelengths of $355 \mathrm{~nm}\left(\mathrm{YAG}\right.$ and $\left.\mathrm{YVO}_{4}\right), 351 \mathrm{~nm}$ 
(YLF), and $349 \mathrm{~nm}$ (YLF). A frequency doubled $\mathrm{Nd} / \mathrm{YVO}_{4}$ as applied to SL will be describe in the following sections.

\section{FREQUENCY TRIPLED Nd/YVO $\mathrm{O}_{4}$ LASER}

$\mathrm{Nd} / \mathrm{YVO}_{4}$ laser has its strongest lasing line at the wavelength of $1064 \mathrm{~nm}$. Frequency tripling of that line results to the wavelength of $354.7 \mathrm{~nm}$. Frequency tripling of a laser beam is a two step process which uses nonlinear optical crystals. It is shown schematically in Fig. 1. The first step is frequency doubling that combines two infrared photons (1064 nm) into a single photon of visible green light $(532 \mathrm{~nm})$. In a doubling process the frequency is doubled and consequently the wavelength is divided by two. The second step of tripling process is frequency mixing as shown in Fig. 1. In this step one green photon (from the doubling process) is mixed with one of the original infrared photons inside another nonlinear crystals. In this process frequencies of the doubled and the original are added up leading to three times the original frequency and to one third of the original wavelength. Fig. 2 shows a practical implementation of the frequency tripling. Original infrared photons (not visible in the photo) arrive from right of the doubling crystal housing. The green photons (visible in the photo) exiting this housing are steered by two mirrors to the mixing crystal housing. All three wavelength (infrared, green, and ultraviolet) photons exit mixing crystal to left (only the green ones are visible in the photo). The infrared and green photons are removed from the beam later by dichroic mirrors.

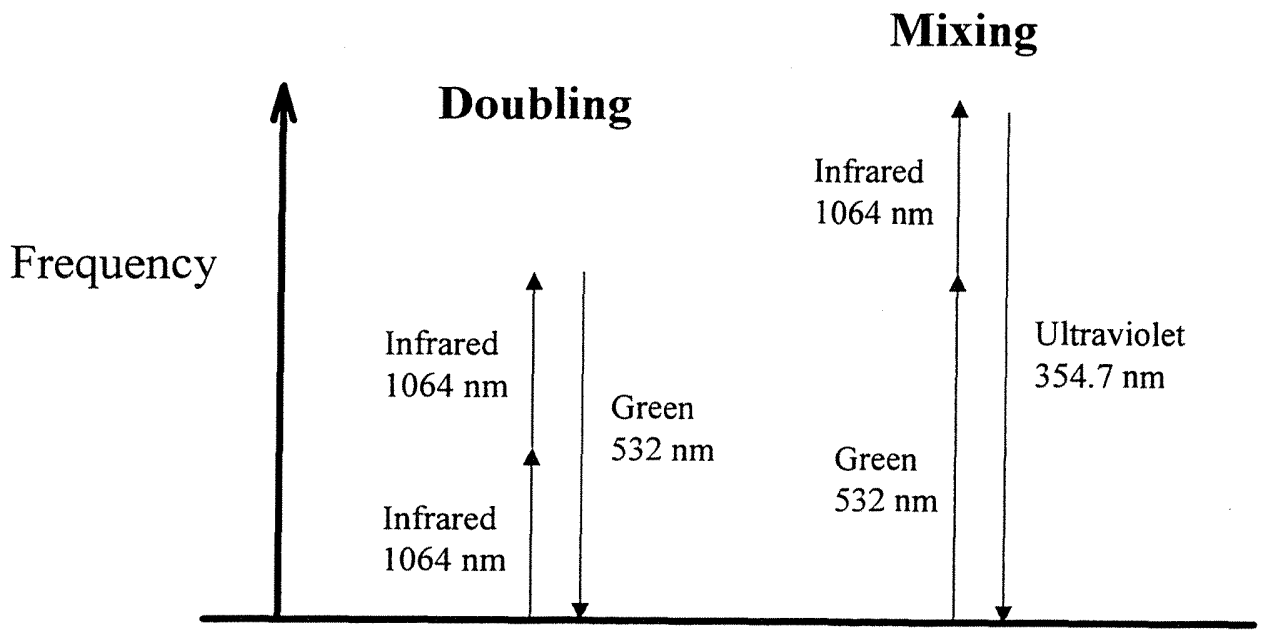

Fig. 1. Schematic diagram of the frequency tripling process. 


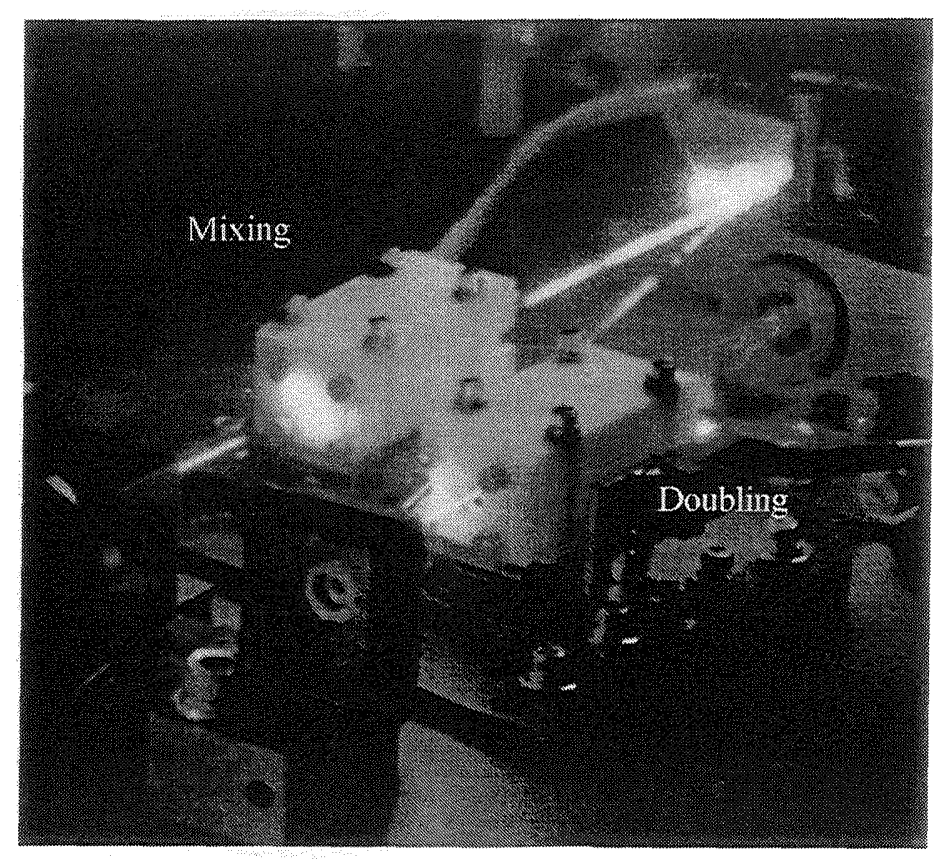

Fig. 2 Photograph of a frequency tripling apparatus.

The nonlinear crystals used in the frequency tripling process require the laser power to be in the range of kilowatts. In order to generate efficiently tens or hundreds of milliwatts of frequency tripled power, the infrared $\mathrm{Nd} / \mathrm{YVO}_{4}$ laser is made with a Q-switched cavity. A schematic diagram of the power output from a Q-switched laser cavity is shown in Fig. 3. The light comes out from the cavity in typically $10 \mathrm{~ns}$ pulses. The period between successive pulses is typically $30 \mu \mathrm{s}$. Thus the power during the short laser pulse can be many thousands times higher than the average power with a Q-switched cavity and therefore the frequency tripling process can be efficient at the infrared laser power level of a few watts.

\section{STEREOLITHOGRAPHY WITH PULSED LASERS}

There are new factors that have to be considered when a pulsed laser is applied to SL, since all previous experience is based on $\mathrm{cw}$ (continuous wave) gas lasers. First, the instantaneous power during the $10 \mathrm{~ns}$ pulse of frequency $\mathrm{Nd} / \mathrm{YVO}_{4}$ laser is more than 3 orders of magnitude higher than the average power or the power of a typical gas laser applied in SL. However, we do not expect any significant optical damage problems at these power levels yet. Also it is questionable if the polymerization process is similar if the same amount of exposure is applied either in $10 \mathrm{~ns}$ or in $30 \mu \mathrm{s}$. Because there are no important chemistry related characteristic time constants in the range from $10 \mathrm{~ns}$ to $30 \mu \mathrm{s}$, we expect that the polymerization process for the repetitive pulsed laser should proceed as if the exposure were applied with a $\mathrm{cw}$ laser. To address this problem we have used a pulsed frequency tripled Nd/YLF laser at $349 \mathrm{~nm}$ in an SLA with SL5180 resin that has designed for Argon Ion laser at $351 \mathrm{~nm}$. The 


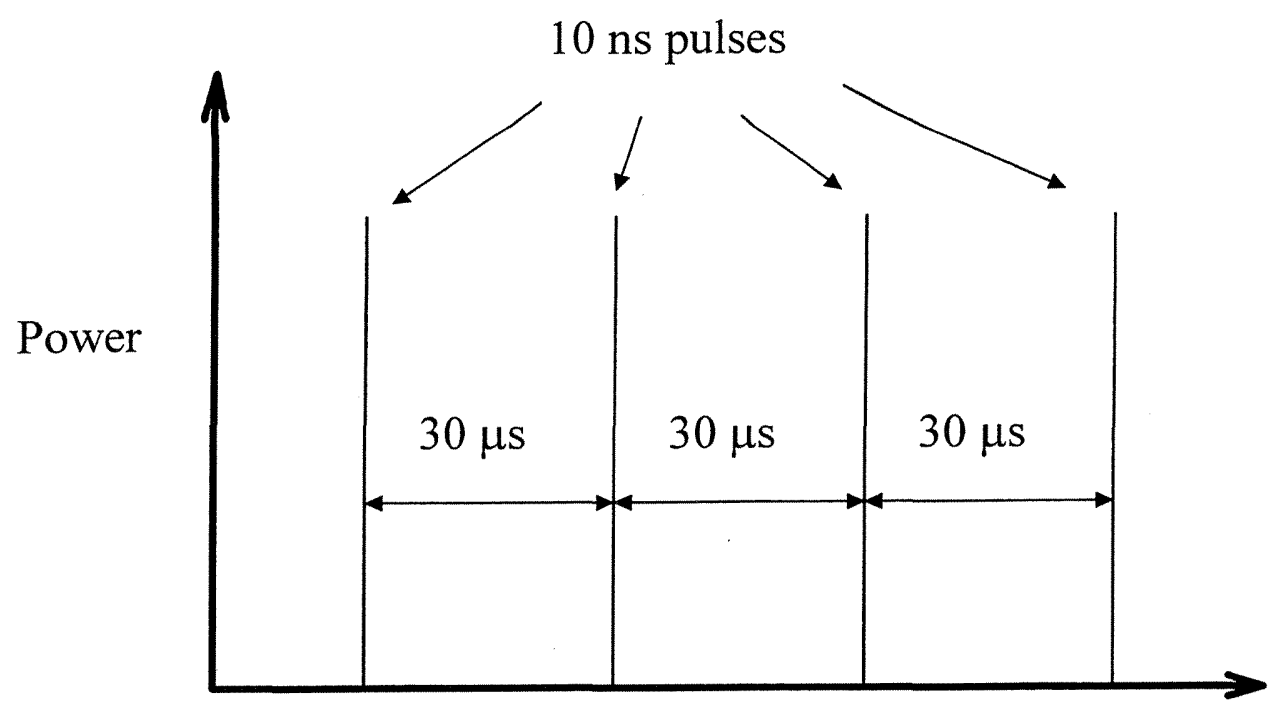

Time

Fig. 3. Schematic diagram of output from a Q-switched $\mathrm{Nd} / \mathrm{YVO}_{4}$ laser cavity.

Windowpanes test [2] results are shown in the Working Curve of Fig. 4. A least mean square fit leads to resin parameters, critical intensity $E_{c}=15.0 \mathrm{~mJ} / \mathrm{cm}^{2}$ and penetration depth $D_{P}=5.0$ mils. These are within experimental error of the default values, critical intensity $E_{c}=16.2 \mathrm{~mJ} / \mathrm{cm}^{2}$ and penetration depth $D_{P}=5.2$ mils, for a cw Argon Ion Laser. On the basis of these Windowpanes and other subsequent experiments, we conclude that we do not observe any significant difference in polymerization if the same exposure is applied either $\mathrm{cw}$ lasers or high repetion rate $(>1 \mathrm{Khz})$ Q-switched pulse laser.

Another factor to be considered is related to scanning speed. In order for the cured line to be continuous and without large modulation, we require the time between the laser pulses to be shorter than the time required to travel laser spot half width $W_{0}$ using the scanning speed $V_{s}$. Based on the analysis presented in Ref. [3] it is straightforward to derive a criteria for the minimum pulse repetition frequency $F_{\min }$

$$
F_{\min }=\frac{V_{s}}{W_{0}}=\sqrt{\frac{2}{\pi}} \frac{P_{L}}{E_{c} W_{0}^{2}} \exp \left(-C_{d} / D_{p}\right) .
$$

Here $P_{L}$ is the average laser power and $C_{d}$ is the expected depth of cured line. In Fig. 5 we plot the minimum repetition frequency $F_{\min }$ as a function of the laser power. We assume the properties of the SL5180 photopolymer resin $\left(E_{c}=16.2 \mathrm{~mJ} / \mathrm{cm}^{2}\right.$ and $\left.D_{P}=5.0 \mathrm{mils}\right)$, the cure depth $C_{d}=D_{P}$, and a spot diameter $2 W_{0}=250 \mu \mathrm{m}$. 


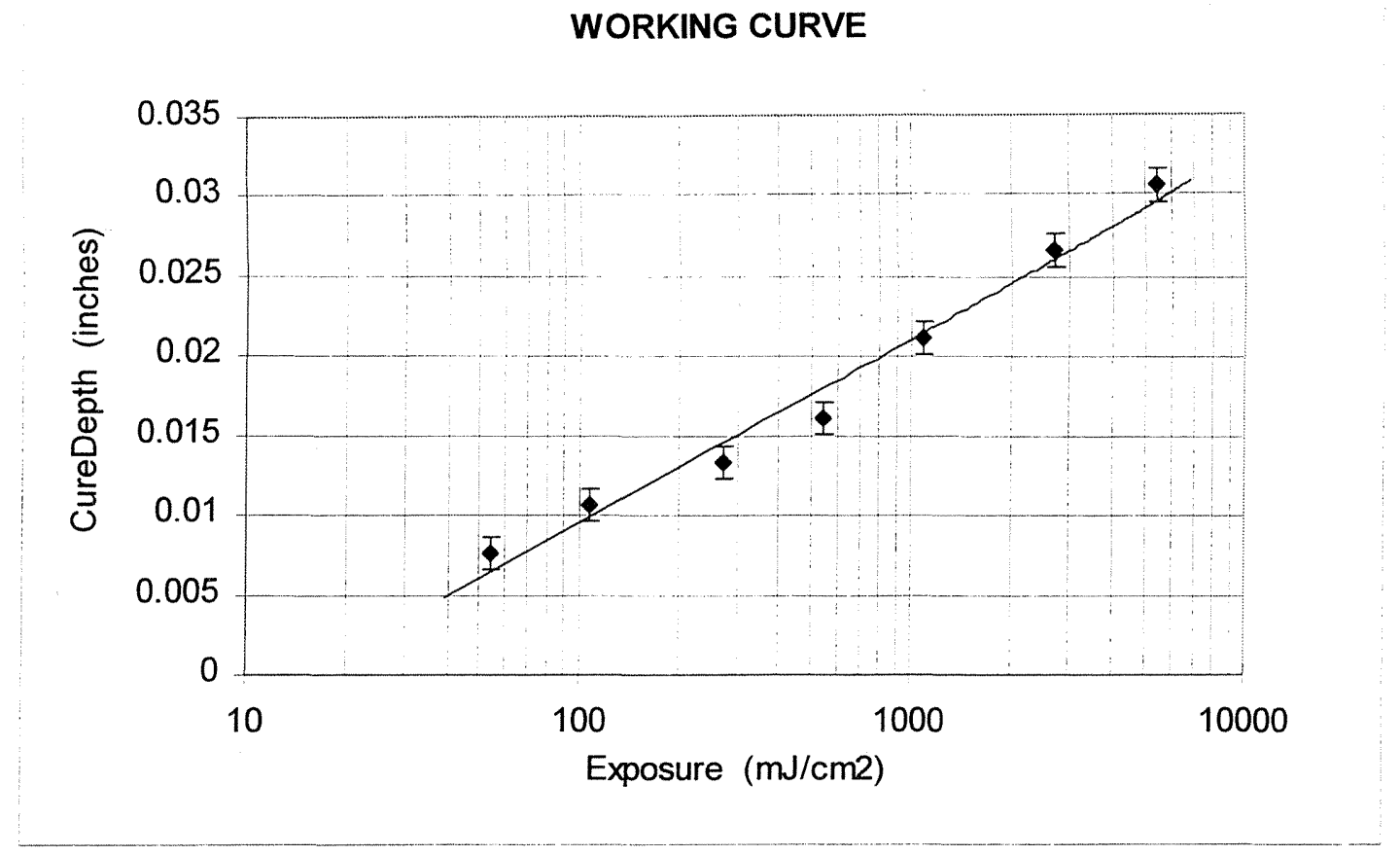

Fig. 4 Working curve for SL5180 resin determined for pulsed frequency tripled Nd/YLF laser at the wavelength of $349 \mathrm{~nm}$.

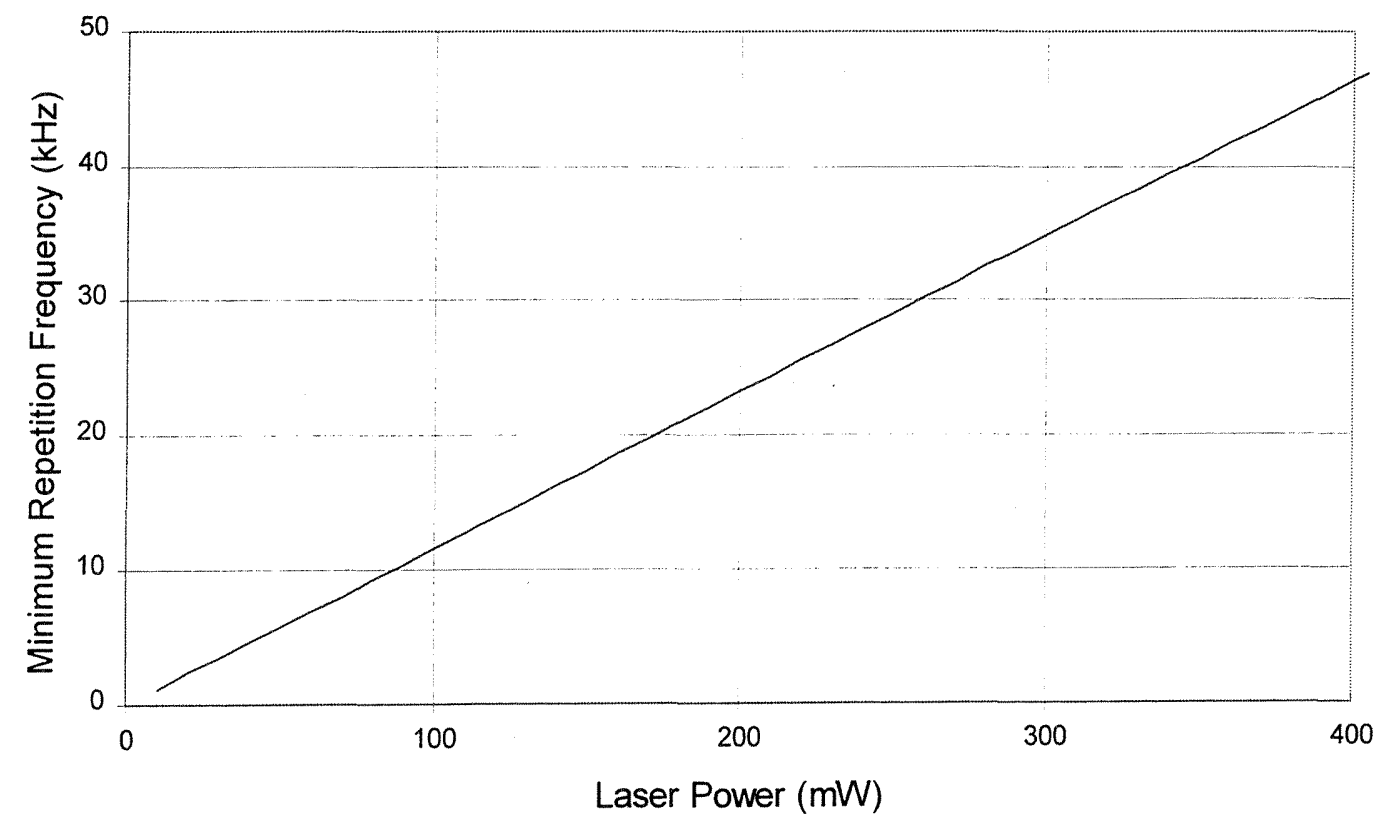

Fig. 5. Minimum Repetition Frequency for a pulsed laser as a function of average power. 


\section{OPTOLASER ${ }^{\text {TM }}$}

It was mentioned in the introduction that diode pumped solid state lasers can be made much smaller than the gas lasers presently used in SL. 3D Systems has taken advantage of this in the development of the frequency tripled diode pumped $\mathrm{Nd} / \mathrm{YVO}_{4}$ laser (OptoLaser ${ }^{\mathrm{TM}}$ ) for the SLA 350. Because the laser cavity itself can be made compact all the beam conditioning optics needed for SL are included in the OptoLaser ${ }^{\mathrm{TM}}$ head. A schematic diagram of the OptoLaser ${ }^{\mathrm{TM}}$ head is shown in Fig. 6. The laser for SLA-350 is manufactured by Spectra-Physics Lasers, Inc. As a result of internal beam conditioning optics, all elements can be factory prealigned with custom tooling.

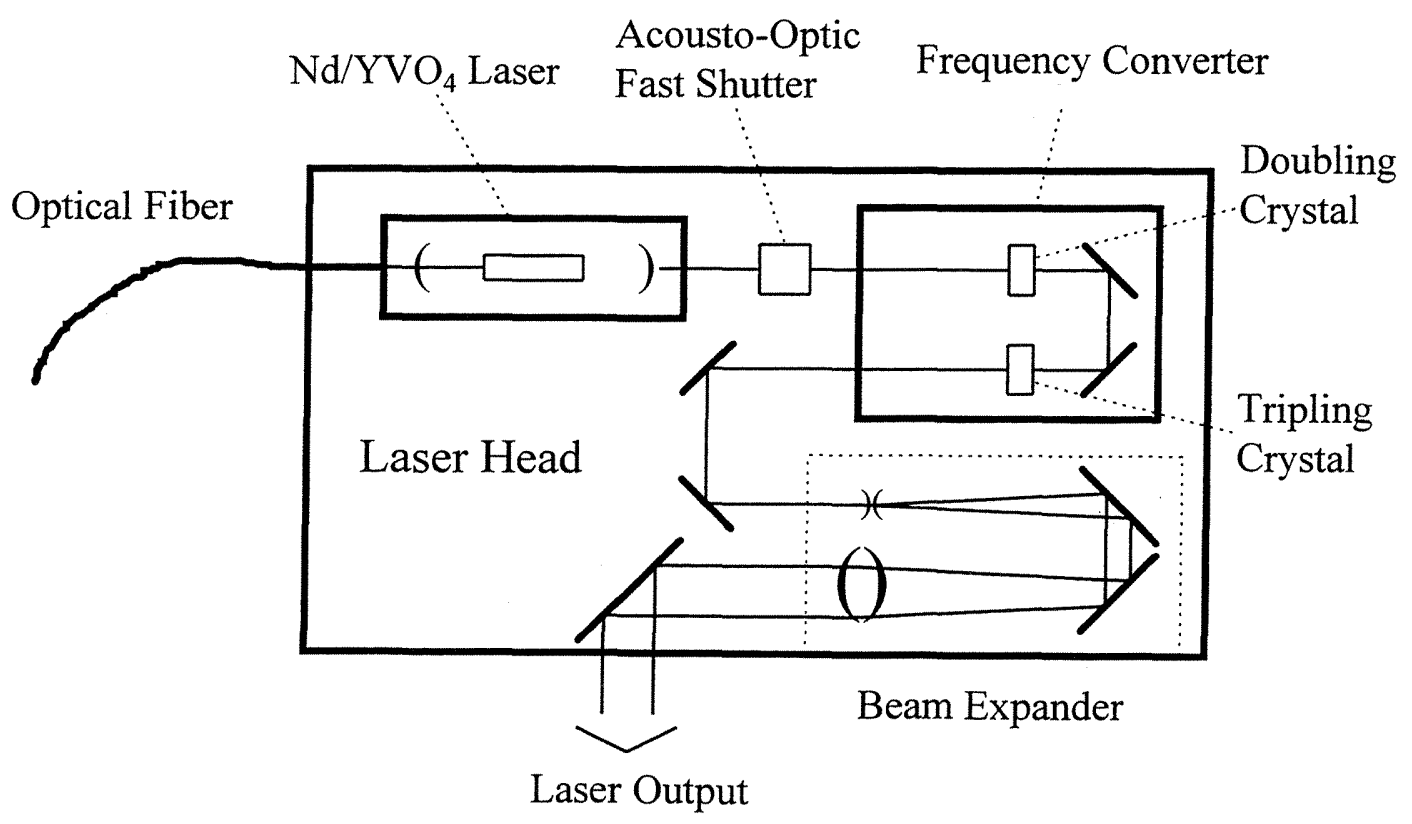

Fig. 6. Schematic diagram of the OptoLaser ${ }^{\mathrm{TM}}$ head used in SLA350.

\section{CONCLUSION}

Ultraviolet solid state lasers have reached maturity level where they can be applied to stereolithography. Their main advantages are: compactness, efficiency, and life expectance. This paper discusses issues that relevant in application of all solid state lasers for stereolithography. 


\section{REFERENCES}

[1] M. Hunziker and R. Leyden, "Basic Polymer Chemistry," Rapid Prototyping \& Manufacturing: Fundamentals of Stereolithography, Ed: P. F. Jacobs. pp. 25 - 58, Society of Manufacturing Engineers, Dearborn, MI, 1992.

[2] H. Nguyen, J. Richter, and P. F. Jacobs, "Diagnostic Testing," Rapid Prototyping \& Manufacturing: Fundamentals of Stereolithography, Ed: P. F. Jacobs. pp. 249 - 285, Society of Manufacturing Engineers, Dearborn, MI, 1992.

[3] P. F. Jacobs, "Fundamental Processes," Rapid Prototyping \& Manufacturing: Fundamentals of Stereolithography, Ed: P. F. Jacobs. pp. 79 - 110, Society of Manufacturing Engineers, Dearborn, MI, 1992. 